\title{
Los límites de la apropiación espacial. La Plaça dels Àngels, Barcelona: un caso de estudio
}

\author{
Os limites da apropriação espacial. A Plaça dels Àngels, Barcelona: um estudo \\ de caso
}

The limits of spatial appropriation. Plaça dels Àngels, Barcelona: a case study

Fran Morente[a] (D)

[a] Universitat de Vic, Vic, Catalunya, Spain

Cómo citar: Morente, F. (2019). Los límites de la apropiación espacial. La Plaça dels Àngels, Barcelona: un caso de estudio. urbe. Revista Brasileira de Gestão Urbana, 11, e20180140. https://doi.org/10.1590/2175-3369.011.e20180140

\section{Resumen}

Ante las distintas tensiones modernas que amenazan el espacio público, este sigue siendo el teatro de representación de la sociedad, tanto para mostrar como para actuar. A todo discurso conceptual urbano hay una respuesta desde la práctica cotidiana del espacio público, la apropiación. Por ello, el objetivo de este artículo es indagar acerca del concepto de apropiación espacial en la Plaça dels Àngels (Barcelona), que representa uno de los espacios públicos más característicos del denominado 'Modelo Barcelona'. A tales efectos, realizamos una investigación cualitativa triangulada, sustentada en el diseño grounded theory, y fundamentada en etnografía y entrevistas. Los resultados muestran que, a pesar de la infradotación de la plaza, existen cinco colectivos diferentes que se apropian del espacio público de un modo muy particular. A través de cada apropiación, el espacio público expresa necesidades no colmadas por los planteamientos urbanísticos actuales y, al mismo tiempo, ofrece una respuesta lúdica de resistencia; dicha respuesta nos lleva a reflexionar sobre los límites de la apropiación.

Palabras clave: Apropiación. Barcelona. Espacio público. Etnografía.

\section{Resumo}

Dadas as diferentes tensões modernas que ameaçam o espaço público, este permanece o teatro de representação da sociedade, tanto para mostrar como para agir. Para todo discurso conceitual urbano há uma resposta da prática cotidiana do espaço público, apropriação. Portanto, o objetivo deste artigo é investigar o conceito de apropriação espacial na Plaça dels Àngels (Barcelona), que representa um dos espaços públicos mais característicos do chamado "Modelo de Barcelona". Para tanto, realizou-se uma pesquisa qualitativa triangulada, baseada no desenho grounded theory e baseada em etnografia $e$ entrevistas. Os resultados mostram que, apesar do infradotation da praça, existem cinco coletivos diferentes que se apropriam do espaço público de uma forma muito particular. Através de cada apropriação, o espaço público expressa necessidades não preenchidas pelas atuais abordagens de planejamento urbano e, ao mesmo tempo, oferece uma resposta lúdica de resistência. Essa resposta nos leva a refletir sobre os limites da apropriação.

Palavras-chave: Apropriação. Barcelona. Espaço público. Etnografia.

FM es doctor em Derecho, Economía y Empresa (UVIC), investigador, e-mail: fjmp.1984@gmail.com 


\section{Abstract}

Given the different modern tensions that threaten the public space, it remains the representational theater of society, both to show and to act. To all urban conceptual discourse, there is a response from the daily practice of public space, appropriation. Therefore, the objective of this article is to investigate the concept of spatial appropriation in the Plaça dels Àngels (Barcelona), which represents one of the most characteristic public spaces of the so-called 'Barcelona Model'. To this end, a triangulated qualitative research was conducted, based on the grounded theory design, and based on ethnography and interviews. The results show that despite the infradotation of the square, there are five different collectives that appropriate the public space in a very particular way. Through each appropriation, the public space expresses necessities not filled by the current urban planning approaches and, at the same time, it offers a playful response of resistance; this response leads us to reflect on the limits of appropriation.

Keywords: Appropriation. Barcelona. Public space. Ethnography.

\section{Introducción}

El objetivo de este artículo es indagar acerca del concepto de apropiación en el espacio público, tomando como caso la Plaça dels Àngels, en Barcelona: buscamos no limitarnos a la descripción de las prácticas cotidianas, sino que, a través de éstas, reflexionaremos sobre las tensiones de amenazan a la ciudad contemporánea.

Sorkin (1992) provoca: ¿está muerto el espacio público? La comodificación (Davis, 1990), la reificación de la experiencia vivida (Lefebvre, 1973), la normativización castradora (Margier, 2017), la desposesión simbólica (Zukin, 1995), la privatización de lo público (Chaplin \& Holding, 1998) son, ciertamente, argumentos a favor. Si el espacio expresa la sociedad (Hiernaux \& Lindón, 1996) y es el lugar donde se crean sus significaciones (Rouet, 2014), el espacio público urbano es síntoma inequívoco de nuestros tiempos (Parkinson, 2012). A tal punto, el significado de espacio público se ha vuelto resbaladizo, tanto en la práctica como en la teoría (Staeheli \& Mitchell, 2007); ha dejado de ser el lugar de simple expresión colectiva, funcional, simbólica, (Madanipour, 1999) y democrática (Parkinson, 2012), el instante urbano de la comunicación total (Choay, 2014), donde los ciudadanos interaccionan libremente (Paquot, 2015), para volverse el teatro de representación par excellence de los conflictos sociales (Ostermann \& Timpf, 2009; Gehl, 2011) y las relaciones de poder (Harvey, 2008). Dado que la estética del espacio público es la ética (Borja, 2004), hay en él un enfrentamiento de distintos relatos de ficción, una disputa por lo real (Rancière, 2015). La hiperinflación de significados y la fragmentación de la ciudad (Ascher, 2004) y la paulatina privatización de estos espacios públicos, antaño democráticos y de solaz (Carmona, 2010), acentuan su carácter público, porque su control define los status y equilibrios de poder en la ciudad (Madanipour, 1999).

Entonces, lo 'público' del espacio público no es apriorístico, sino que se conquista a través de la práctica cotidiana, porque si un espacio público no es practicado, no merece ser llamado como tal (Paquot, 2015). Estas prácticas deben inscribir las experiencias polisémicas de la ciudad, en contra de la uniformización, ensanchar los límites de la sociedad y, sobre todo, dar cabida a la diferencia (Margier, 2017), porque los conflictos (perniciosos) no radican en las diferencias, sino en las distancias (Massey, 2005).

El ciudadano no ha de comportarse como un mero consumidor de espacio, sino que ha de convertirse en un practicante en el espacio, en un hacedor. En este contrataque contra la pasividad, Harvey (1973) añade una nueva acepción al siempre maleable concepto de imaginación geográfica [geographical imagination]; sostiene Harvey que esta imaginación vincula a los individuos, las comunidades y las organizaciones a sus espacios y a sus dinámicas visibles e invisibles, a múltiples escalas; supone el reconocimiento de los lugares como como asideros biográficos como escenario de flujos transaccionales de diversa índole y, por encima de todo, manifiesta diferencias y similitudes espaciales que, potencialmente, pueden provocar alianzas o confrontaciones por la justicia espacial. Con la imaginación 
geográfica el espacio cobra un significado polisémico, una práctica espacial en que la opresión y la libertad se rozan constantemente.

Las limitaciones de uso espacial ocultan tensiones sociales y conflictos, y estas 'orientaciones prácticas' que emanan de los choques de poder se manifiestan con especial virulencia en los usos del espacio público (Bassand, 1990). Detrás de todo uso hay una 'política de la pasividad', que menoscaba el potencial creador de los cuerpos (Chaplin \& Holding, 1998). El espacio público no es territorio virgen y libertario, al contrario; se trata de un espacio que precisa conquistarse derribando los falsos conceptos de espacialidad [spatiality] que limitan las construcciones sociales que en él pueden tener lugar (Massey, 2005). Según Lefebvre (1974), por inscribirse existencialmente en el espacio, este campo de batalla en el interior de las ciudades tiene un nombre, la producción del espacio [production de l'espace], y unas estrategias ciudadanas, la apropiación [appropiartion].

El concepto lefebvriano de apropiación aúna teoría y praxis. Distingue 'hábitat', la morfología aislable del espacio y físicamente localizable, del 'habitar', acción de los cuerpos vivos, libres y creadores. A esta búsqueda creadora de espacios habitables lo denomina apropiación, la fuerza emancipadora, la inscripción del deseo, el acto revolucionario y creador de los ciudadanos en los espacios urbanos (Lefebvre, 1973). Al grabar los cuerpos en los dinámicos tempos urbanos, los ciudadanos convierten la ciudad en obra de arte colectiva (Lefebvre, 1968). Mediante la apropiación los espacios son utilizados en su sentido más amplio y democrático (Abrahao, 2016); la alternancia, la alteridad y la pluralidad de las prácticas sociales dan lugar a usos alternativos del espacio que responden a necesidades e intereses concretos (Lefebvre,1974). De este modo, la dialéctica marxista de Lefebvre (1974), que contrasta 'espacio planeado' por arquitectos y urban planners con 'espacio vivido', practicado y subjetivamente colectivo, halla en la apropiación la articulación el espacio en tanto sujeto activo; ya no se trata de acontecimientos que suceden en un espacio instrumental, sino que el espacio crea acontecimientos (Soja, 1996).

Más práctico, Bassand (1990) simplifica: apropiación es la expresión espacial de los ciudadanos, que es resultado de las relaciones de poder y las necesidades de los colectivos que se enzarzan en la producción del espacio. Este encuentro entre usos y símbolos que se solapan, se suman y anulan en el espacio, genera interacciones inesperadas en el espacio público, el cual se convierte en el escenario de disputa de poder (Madanipour, 1999). El concepto ha gozado de aceptación académica, especialmente en Estados Unidos donde Lefebvre ha sido redescubierto (Morente, 2018), para emplearse desde diversos ángulos: geográficos, sociológicos, antropológicos; algunos casos recientes son: la apropiación en espacios públicos brasileños (Abrahao, 2016), ritos religiosos de inmigrantes filipinos en espacios públicos italianos (Saint-Blancat \& Cancellieri, 2014), la exploración de espacios pseudopúblicos como supermeracados, centros comerciales y carreteras (Mierzejewska, 2011) o, incluso, el análisis espacial de las apropiaciones en parques a través de los GIS (Ostermann \& Timpf, 2009). No obstante, y es aquí una de las carencias que queremos subsanar, no se ha estudiado la contraposición entre el uso conceptual, esperado y oficial y el uso real y vivido. Partiendo de este 'gap', ahondamos en la apropiación, porque a través de ella el espacio abandona el reino de Lo Abstracto por el de Lo Vivido (Lefebvre, 1974): es la révolution de l'espace. Siendo a un tiempo un uso físico y simbólico (Lefebvre, 1974), la apropiación espacial es la materia prima del capital simbólico urbano (Zukin, 1995). Vidal \& Pol (2005) distinguen en la apropiación lefebvriana, por una parte, la creación de espacios simultáneos y habitables según el deseo y, por la otra, la identificación y el sentimiento de pertenencia simbólica. Como expone Margier (2017), retomando la distinción hábitat-habitar, la apropiación consiste en una acción de transformar un espacio en un hogar, la 'constitución de un hogar' [constitution d'un chez-soi].

La apropiación es el camino hacia la conquista espacial, y es el concepto principal de este artículo. Para investigar estas tensiones representacionales en los espacios públicos urbanos, Barcelona se presenta como un caso de estudio muy interesante y del que se pueden extraer varias lecciones futuras. En los últimos cincuenta años, Barcelona ha experimentado una profunda transformación urbana, atribuible a una forma muy sui generis de entender lo urbano (Benach, 1997; Capel, 2005; Casellas, 2006). 
Tras las dos Exposiciones Internacionales -respectivamente, 1888 y 1929-, que cambiaron para siempre la fisonomía de Barcelona, ésta se renovó definitivamente a finales de los setenta de la mano del Plan General Metropolitano. Este fue el nacimiento del 'Modelo Barcelona', cuyos efectos se han hecho sentir a lo largo y ancho de las siguientes décadas (Capel, 2005). Durante los años ochenta, y dejando atrás años de estancamiento franquista, el Ajuntament de Barcelona comenzó una importante rehabilitación de los espacios públicos, afectando a su diseño y a su función orgánica (Bohigas, 1986). Esta orientación pasaba por convertirlos en lugares de encuentro local que solucionaran problemáticas como la sobrecongestión urbana, fueran núcleos de reunión, articularán el tráfico a pie y, esencialmente, revertieran el aislamiento de ciertas zonas de la ciudad (Capel, 2005). Con la designación olímpica en 1986, Barcelona sufrió su mayor metamorfosis urbana hasta el momento (Benach, 1993; Reventós Gil, 2007). Barcelona '92 puso en bandeja el pretexto de llevar el 'Modelo Barcelona' hasta su máximo esplendor. El Pla Estratègic Barcelona 2000, de 1990, fue, y casi citamos verbatim, la 'herramienta institucional' para llevar a cabo estas grandes transformaciones, cuya última intención no era otra que convertir Barcelona tanto en una ciudad de calidad urbana como centro económico de la región (Reventós Gil, 2007). Se iniciaban entonces las actuaciones 'minimalistas', con efectos sobre la revalorización de los barrios -entre ellas, la integración y dignificación del Raval, que veremos en próximas páginas, y 'las estéticas', con ánimo de crear un capital cultural y simbólico referencial. En pocas palabras, un urbanismo ambicioso que, aprovechando el empuje de grandes acontecimientos, combinaba actuaciones sociales en los espacios públicos locales con el posicionamiento internacional de Barcelona como epicentro cultural y económico (Rius \& Sánchez-Befando, 2015). Los dos modos antonomásicos fueron las 'metástasis selectivas', concepto acuñado por Oriol Bohigas, uno de los más importantes arquitectos de este nuevo urbanismo, y el 'esponjamiento' (Capel, 2005). Por el efecto metastático cabe entender intervenciones urbanas muy puntuales, mayormente a través de la monumentalización de espacios públicos -esculturas de artistas prestigiosos o diseños urbanos de vanguardia- ubicados en barrios esquilmados, para crear un efecto regenerador en los aledaños y de atracción de capital. El esponjamiento supuso la renovación de infraestructuras culturales en puntos concretos para ahondar en la conexión entre los barrios desfavorecidos con los flujos económicos de la ciudad (Benach \& Tello, 2004).

Así, el 'Modelo Barcelona' no cabe entenderlo sólo como una forma de actuar sobre la ciudad sino como toda una declaración teórica de intenciones (Benach, 1997). Casellas (2006) resume eficazmente el modelo en seis puntos: (1) participación del sector privado en la financiación de proyectos urbanos; (2) creación de entidades autónomas para dirigir el proceso de planeamiento urbano, su diseño y gestión; (3) consenso entre administraciones públicas; (4) introducción de planificación estratégica; (5) utilización de grandes acontecimientos y la cultura como estrategia transformadora; y (6) participación ciudadana.

Hasta principios de los años 2000, el consenso era abrumador: el modelo había sido un éxito (Subirós, 1999). No obstante, a raíz del Fòrum de les Cultures, en 2004, el modelo empezó a presentar alarmantes signos de agotamiento (Rius \& Sánchez-Befando, 2015) y, en palabras de Capel (2005), una obsesión nada sana por la modernidad. Las críticas no se hicieron esperar. A la larga, el modelo había propiciado una gentrificación irreversible (Benach \& Tello, 2004) que había yuppificado la ciudad (Capel, 2005) y convertido su urbanismo en pura mercadotecnia turística (Rius \& Sánchez-Befando, 2015). En su perversión, el modelo en su agotamiento estetizante se había convertido en la 'Marca Barcelona' (Reventós Gil, 2007).

En los últimos años, con la renovación del último tramo de la Diagonal, que se extiende de Glòries al 22@, y la apuesta política por las Superislas, cuyo primer pilot test está en el barrio de Poble Nou, el urbanismo barcelonés busca una alternativa al 'Modelo Barcelona'.

El caso que abordamos en este artículo, la Plaça dels Àngels, es una de las intervenciones más paradigmáticas y emblemáticas del 'Modelo Barcelona', de ahí su interés: en este espacio público se sintetiza los límites de dicho modelo; y en su apropiación, resuena un mensaje que no debemos silenciar. 


\section{Metodología}

En un objeto de estudio abierto y sincrónico como el espacio público, la metodología empleada siempre planteará problemas de captación, aprehensión, análisis y síntesis (Joseph, 1988). Dado que la percepción geográfica de las personas está saturada del subjetivismo (Andrés Sarasa, 1991), optamos por una metodología cualitativa, ideal para captar y tratar el dato vivo (Yin, 2011), y la basamos en el diseño Grounded Theory. Las fases fueron:

Fase 1. Exploración. Para familiarizarnos con la Plaça dels Àngels, utilizamos dos métodos. El primero de ellos fue la investigación documental fue de dos tipos: (1) investigación documental sistemática, que incluyó datos, estadísticas, informes, papers, guías turísticas, blogs, fotografías, noticias, proyectos y planes urbanísticos de Barcelona (ciudad), el Raval (barrio) y la Plaça dels Àngels (el caso); y (2) indagación especializada en espacio público con vistas a conseguir un marco teórico amplio y maleable. Esta indagación proporciona el punto de partida (Yin, 2011). El segundo método fue la observación flotante [observation flottante] (Pétonnet, 1982), basada en entrar en el campo de estudio con la atención abierta, sobre nada concreto y anotando las impresiones a vuelapluma y dibujando o fotografiando los situaciones que allí tienen lugar. Este primer contacto físico con el caso empezaba a comunicar la bibliografía con el dato vivo. La Fase 1 se extendió de enero a mayo de 2017.

Fase 2. Convergencia. La captación del dato cualitativo se realizó con dos métodos interconectados. El más laborioso fue la etnografía; según Hammersley \& Atkinson (2007), los hechos existen tamizados por la experiencia fenomenológica, y es preciso observarlos desde dentro de la muestra-emic- para que aflore su modo particular de entender el mundo. Puesto que el dato etnográfico y su contexto son uno, y para no romper el naturalismo y contaminar el dato buscamos convertirnos, en la medida de lo posible, en invisible y no intrusivo (Agar, 1996). La observación estructurada tuvo lugar entre dos y tres veces a la semana, en horario diurno -en ocasiones la geografía diurna y nocturna no tienen nada que ver entre sí (Joseph, 1988)-, de mayo a octubre. Atendiendo a los 'microlugares' [microsettings] (Duneier, 1999), fijamos en el Cuaderno de Campo (Cuaderno, en adelante) las posiciones de los distintos grupos, sus movimientos, sus interacciones y las variaciones, que nos dieran información sobre su apropiación, el posible determinismo arquitectónico y los límites sociales. Asimismo, tomamos fotografías y registros sonoros. A este método, a partir de septiembre y hasta octubre, y tras alcanzar la saturación teórica [theoretical saturation] (Glaser \& Strauss, 1967), introdujimos el segundo método, entrevistas de intercepción [intercept interviews] (Schneider, 2013); acercándonos a 53 participantes, les abordamos en frío para realizar, con su consentimiento, una entrevista cualitativa semiestructurada, cuyo objetivo era conocer hábitos, opiniones e historias personales en la plaza, porque estas mismas historias son una forma de conocimiento (Seidman, 2006, p. 7). En octubre dimos por concluida esta Fase.

Fase 3. Análisis y síntesis. Los heterogéneos datos captados en las dos anteriores fases -bibliografía y datos fenoménicos- alimentaron el diseño de grounded theory, los cuales los codificamos -un proceso iterativo que supuso 9 versiones- para crear, paulatinamente, categorías agregadas y patrones que, al relacionarlos, nos ofrecían potentes hallazgos explicativos (Glaser \& Strauss, 1967) sobre las estrategias de apropiación espacial en la Plaça dels Àngels. Esta última fase se extendió de octubre de 2017 a marzo de 2018.

\section{Caso de estudio: Plaça dels Àngels}

Nuestro caso de estudio es la Plaça dels Àngels, que se trata de un ejemplo paradigmático del 'Modelo Barcelona', ya que aglutina la intervención del espacio público mediante la monumentalización, la metástasis selectiva y la instrumentalización de la cultura para promocionar internacionalmente la ciudad. Además, esta plaza posee una ubicación excelente; es un intersticio entre antagónicas condiciones de los del Raval y l'Eixample; un cruce de caminos entre uno de los barrios con mayor tasa de inmigración, económicamente deprimido y de intrincada trama urbana con otro turístico, comercial y emblema de la Barcelona progresista de Ildefons Cerdà y desbordado por el turismo. 
Estas dos condiciones convierten nuestro caso de estudio en una paradoja viviente. La actual Plaça dels Ángels tiene su primera piedra de toque en el Pla Especial d'Ordenación del Conjunt de la Casa Caritat (PE, en adelante), a partir del cual se deseaba reformular el norte del Raval como cuna cultural de Barcelona, y su epicentro estaría delimitado por las calles de Joaquim Costa, Valldonzella, Montalegre y Ferlandina (Barcelona, 1990). La implantación de edificios con una nueva actividad cultural ya había sido ensayado con éxito en Le Marais en París, el Vieux Port de Marsella, la City de Londres y las zonas portuarias de San Francisco y Boston (Barcelona, 1990).

La actuación combinó la rezonificación de usos, la creación de espacios públicos, la descongestión urbana y, sobre todo, la rehabilitación de activos y la creación de nuevos equipamientos culturales con ambición de proyección internacional, una concentración que algunos autores (Subirats \& Rius, 1998) han denominado 'Clúster Artístico de la Plaça dels Àngels'. En la necesidad de posicionar a Barcelona como referente urbanístico, y en una importante apuesta por la economía de escala (Barcelona, 1990), los equipamientos culturales que conforman el clúster fueron el Centre de Cultura Contemporànea de Barcelona (CCCB), el Museu d'Art Contemporani de Barcelona (MACBA), el Hotel d'Entitats, Auditori y Centre d'Arts Audiovisuals, que acompañan la remodelación del Pati Maning y del Convent dels Àngels y la nueva ubicación de la Facultat de Geografia i Història de la Universitat de Barcelona. Y en mitad del clúster, con $4.040 \mathrm{~m}^{2}$ de superficie y un coste de 80,8 millones de pesetas, la Plaça dels Àngels (Barcelona, 1990). Inaugurada en 1995, la plaza constituyó una de las más importantes actuaciones; considerada por el ayuntamiento como la plaza mayor del Raval, la Plaça dels Àngels (Figura 1, Figura 2, Figura 3) fue renovada para (1) repercutir positivamente en la vida de los habitantes, esponjar la densificación urbana, conectar y regenerar el Raval y (2) convertirse en el punto desde donde contemplar el diseño arquitectónico del MACBA -que recayó en Richard Meier, un lecorbusiano (Montaner, 2003)- y dar cabida a actividades de atractivo internacional que contagiasen el contorno urbano (Barcelona, 1990; Benach \& Tello, 2004). Una plaza dura, amplia, abierta, desprovista de vegetación y dotada de un parco mobiliario urbano que deja a las claras su principal función, realzar la arquitectura del MACBA (Pujades, 2002). Así la Plaça dels Àngels es percibida como una muestra del reverso negativo del 'Modelo Barcelona', donde se perdió la oportunidad de solucionar la segregación socioespacial porque se prefirió la impostura estética, en busca de capital internacional (Tapia, 2007); una nueva contribución a 'situar a Barcelona en el mapa' pero a costa de sacrificar el bienestar de los ciudadanos (Benach \& Tello, 2004).

Al delinear su genealogía espacial, comprendemos que la Plaça dels Àngels es un espacio público y, al mismo tiempo, mucho más.

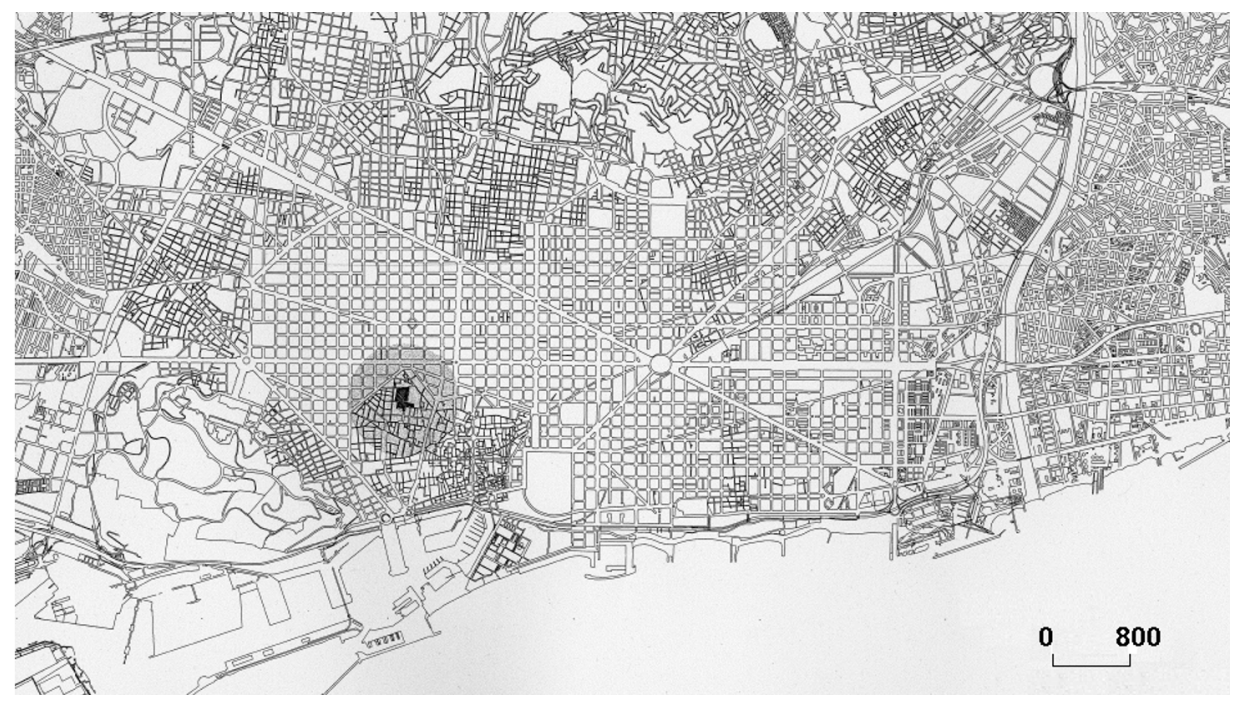

Figura 1 - Situación de la Plaça dels Àngels (en metros), escala original 1:40.000. Fuente: Barcelona (2006). 


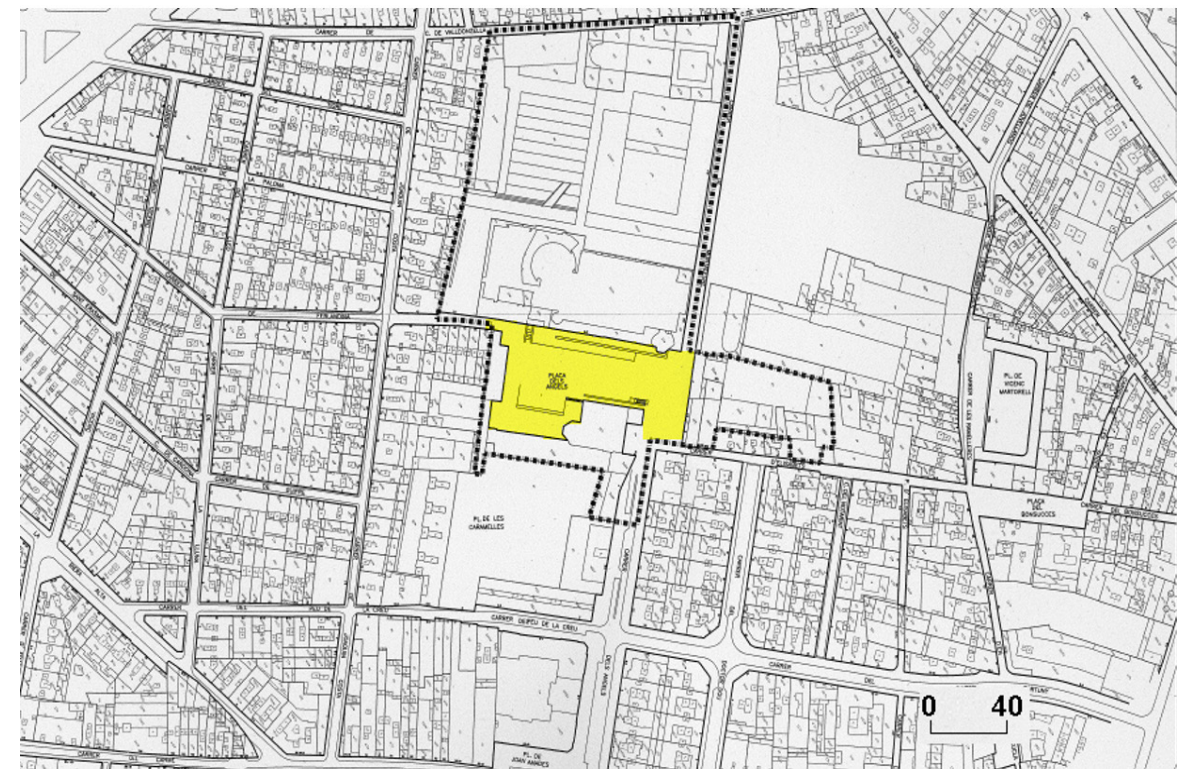

Figura 2 - Situación de la Plaça dels Àngels (en metros), escala original 1:2.000. Fuente: Barcelona (2006).

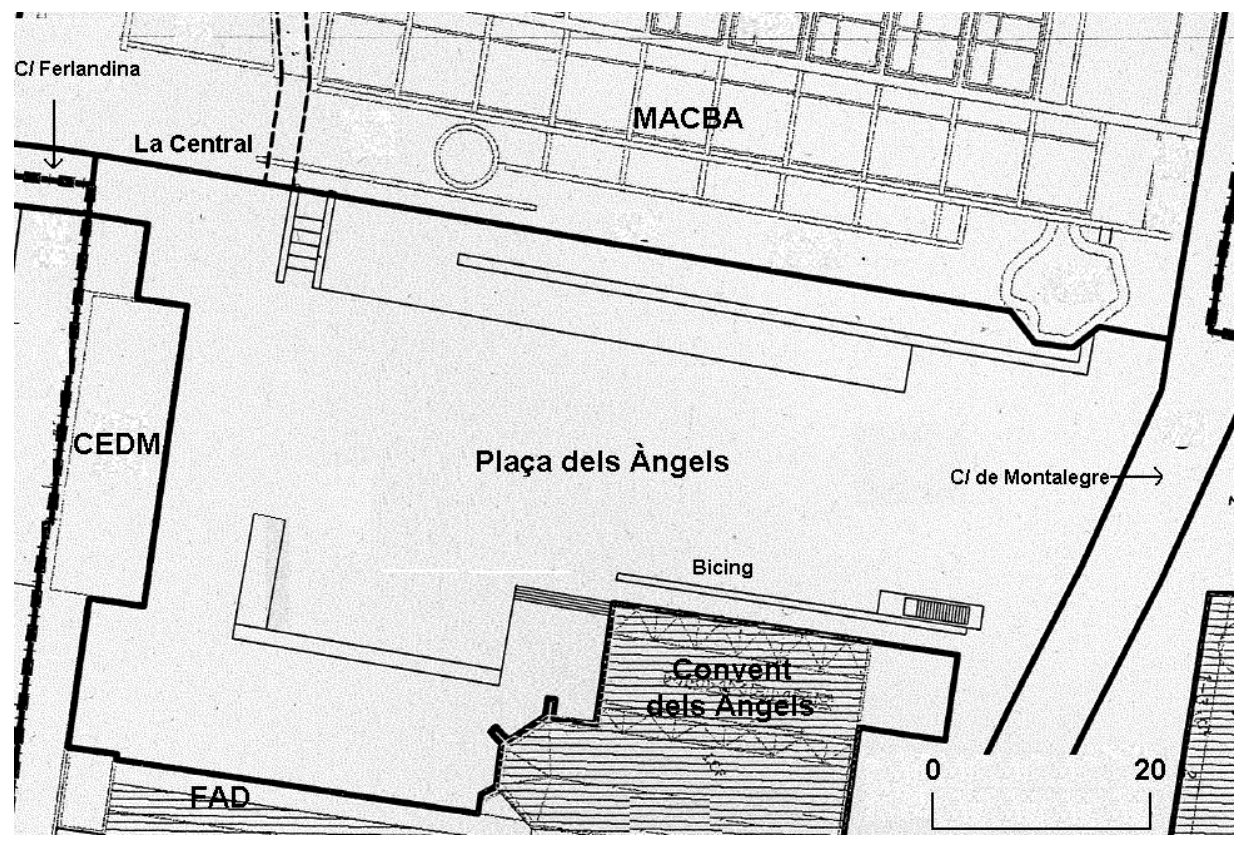

Figura 3 - Situación de la Plaça dels Àngels (en metros), escala original 1:1.000. Fuente: Barcelona (2006).

\section{Resultados y discusión}

La afirmación de Zukin (1995), por la cual la ciudad ideal proyectada por los arquitectos nunca influye sobre la ciudad real, es falsa. Lefebvre (1974) lo entendió al distinguir tres dimensiones en la planificación urbana: planificación material -amoldar la dimensión física del espacio-, planificación financiera -transformar el simbolismo espacial e insertarlo en el segundo circuito del capital- y planificación espaciotemporal -organizar la vida cotidiana-. La fuerza del urban planning recae en su capacidad de moldear el espacio y de crear discursos dominantes que cierren la entrada a nuevas imaginaciones (Choay, 2014), que depreden otros usos espaciales (Wittmer, 2017) al denostarlos como poco aceptables (Iveson, 1998).

Y ahora lo comprobaremos. 
La Plaça dels Àngels conecta la calle de Ferlandina con la de Montalegre, esto es, sutura la enrevesada trama del Raval con la modernismo del Eixample. La gran extensión vacía de esta plaza dura, sin zonas verdes ni refugio durante las horas de insolación, invita a atravesarse sin gusto, a apenas vivirla como un interludio. Si cruzamos la plaza desde Ferlandina, uno se topa con la fachada de la Escola Vedruna-Àngels y el camino se bifurca en tres direcciones; si, por el contrario, venimos de Montalegre, la presencia de las rampas del museo obliga a modificar la trayectoria de nuestros pasos. De entrada, la plaza se presenta como un no-lugar [non-lieu] (Augé, 1993): “Un espacio transitivo, no antropológico, donde la inscripción social es apenas realizable" (Cuaderno, 15/06/2017). A lado y lado de la senda, las referencias visuales polarizadas del Convent dels Àngels y el MACBA, lo antiguo y lo rabiosamente moderno. Ante la infradotación de elementos de reunión, como observamos durante la etnografía, el MACBA (Figura 4) atrae la mirada de los transeúntes, que repercute en la percepción mental de la plaza. En el interior de la plaza, además del omnipresente gris, el servicio de bicis -Bicing- y la entrada al parking acaban de ofrecer, secundariamente, el resto de referencias visuales:

Una vez dentro, no hay horizontes; nuestra mirada atenazada, nuestro alcance visual reducido. No hay orientación, salvo por las dos salidas que impelen a transitar, no a habitar (Cuaderno, 31/05/2017).

La plaza podría estar ubicada en cualquier punto de Barcelona; su contextualización con el entorno circundante es nula. Su diseño actúa de esponjamiento urbano, induce al tránsito constante y a la admiración del edificio de Meier; otros usos parecen excluidos. Diríamos que su imagenabilidad [imageability], su capacidad de evocar y de excitar la imaginación urbana (Lynch, 1960), es exigua: "Poca singularidad y continuidad, escasa diferencia y alcance visual, carencia de nombres y significados" (Cuaderno, 01/09/2017). Si dependiese su diseño, la pasión por la improvisación urbana serían pocas.

No obstante la estructura urbana no siempre coincide con la experiencia urbana (Amendola, 2000). Dado que las instituciones no han 'sacado la cultura a la calle', los ciudadanos, por iniciativa propia, han practicado la Plaça dels Àngels como lugar de representación, desbordando las proyecciones iniciales; la plaza ha sido reclamada por actores secundarios y figurantes del 'Modelo Barcelona' (Benach \& Tello, 2004). La plaza ha sido apropiada, y nuestra etnografía no hizo más que constar esta apropiación que introduce, respecto a la proyección inicial desvíos - detournement situacionista, la reutilización sardónica de elementos prexistentes, la desviación respecto a los usos y significados 'normales' (Trier, 2014).

En la plaza hallamos dos movimientos. Por un lado, el flujo constante de peatones (Figura 5) que ocurría en la zona central de la plaza, y donde se cumplía la función principal de la plaza de conectar los dos barrios. Por el otro, el más interesante, la apropiación de microlugares por distintos colectivos. La apropiación del espacio público responde a una corrección espontánea de las ineficiencias urbanas (Bassand, 1990), y la apropiación de la Plaça dels Àngels manifiesta la necesidad de un lugar de encuentro. Esta apropiación la llevan a cabo distintos colectivos con estrategias de apropiación particulares; veámoslos.

Uno: 'Familias'. Conformado por padres relativamente jóvenes, de 30 a 40 años, con niños que, en muy pocos casos, excedían los 10-12 años (Figura 6). Este colectivo aparecía a las cinco de la tarde y desaparecían a las ocho y media, e, invariablemente, se ubicaban en la primera rampa. Los requisitos de la elección del lugar parecían responder a un suelo limpio:

La plaza es muy rollo Barcelona... está más pensada para los de fuera [turistas] que para los que vivimos aquí... Ni columpios, ni toboganes. Está un poco desangelada, pero menos da una piedra. Al menos está cerca de casa (A, 15/09/2017).

A pesar de su infradotación, utilizaban la plaza por su cercanía respecto a sus hogares: "No es perfecta. Está cerca. Tampoco hay muchos sitios donde llevar a los críos. Es ruidosa. Me siento aquí [en la rampa] y como es amplia puedo ver tranquila al niño" (B, 28/09/2017). Que dentro de la plaza posean una visión general de la misma, les tranquila porque les permite vigilar al niño, a pesar de que las actividades quedan muy circunscritas: "Si traigo a mi hija con los patines se lo pasa bien. Pero jugar a otra cosa no. Por las tardes hay demasiada gente" (C, 19/10/2017). Dentro de este colectivo, los niños venían a veces acompañados por los abuelos, a quienes les desagradaba la plaza: "Demasiado ruido... mucha gente. No 
estás tranquilo. Los del monopatín no tienen respeto por nadie" (D, 05/09/2017); o: "Huele a porro. Hay gente rara. Ponen música con el cacharrito [smartphone]. Me pone nervioso. Vengo por la nieta, que si no... Al menos hay sombra" (E, 08/09/2017). Por lo general, la plaza les parecía poco acogedora por las características físicas y por los colectivos restantes, pero ante la necesidad lúdica del hijo/nieto, consumaban su desvío: acudían a ella por tratarse de lo más próximo.

Dos. 'Jóvenes'. En este colectivo identificamos estudiantes de las dos universidades próximas (Univeristat Pompeu Fabra y Universitat de Barcelona) y gente joven, la mayoría, procedente de otros barrios (Figura 7).

La tienes al lado de la uni [versidad]. Hay un montón de espacio. Si somos muchos es un buen sitio para pasar la tarde. $O$ cuando sales de un examen para relajarte. El ambiente, la gente... es, no sé, guay (F, 14/10/2016).

A diferencia del colectivo anterior, destacaban el ambiente 'moderno' de la plaza y la cantidad de espacio para sentarse en el suelo y escuchar música, hablar o relajarse:

El ambiente mola. Con los skaters... gente con rollo alternativo. De vez en cuando montan espectáculos ahí [señala la esquina del Convent dels Àngels] que son chulos (G, 07/09/2016).

Era el colectivo más disperso; se ubicaban en las rampas centrales, mezclados con las familias, pegados a la fachada del museo e, incluso, en el otro extremo, aprovechando las escaleras o el único banco. Valoraba una comodidad y un ambiente diferente al de las familias. Los jueves y viernes, a última hora de la tarde, no era raro verlos consumir alcohol. Dada su proximidad con el ambiente alternativo del Raval, la plaza atraía a jóvenes de otros barrios: "Venimos de [l barrio de] Gràcia. Está cerca del Raval, de Tallers... Hay un ambiente joven. Pasamos un rato aquí, después vamos a hacer unas birras... Merece la pena" $(\mathrm{H}$, 27/10/2017). Lejos de tratarse de un acto de rebeldía, su apropiación era gozosa y 'desviada'. Lo que algunos considerarían 'marginal' o 'peligroso', este colectivo lo consideraba 'alternativo', y poseía el magnetismo de una curiosidad por saciar.

Tres: 'Turistas'. La estancia de este colectivo (Figura 8) en la plaza se ceñía a media hora como máximo; tomaban la plaza como un lugar donde recobrar el ánimo después de sus caminatas: "Por nada en especial. Estamos cansado. Hace mucho calor. Y aquí hay la sombra" (I, 16/09/2017). Solían sentarse en las rampas de acceso al museo, evitando a los sintecho y fuera de la trayectoria de los skaters. Sin una motivación especial por visitar el museo, aprovechan para retratar el MACBA desde diversos ángulos y llevarse un recuerdo: "Un edificio muy bonito. Llama la atención... Ese blanco" (J, 28/09/2017). Dada su presencia circunstancial, algunos bares aprovechaban para promocionar brunchs y tapas. De entre todos los colectivos, éste era el único no proponían ningún desvío respecto a lo normativo. La plaza era un pasaje entre dos zonas y el punto idóneo para retratar un edificio que proponía a Barcelona como adalid de la Modernidad.

Cuatro: 'Sintecho'. En su mayoría hombre, pasan gran parte del día en la sombra (Figura 9); algunos beben cervezas, otros juegan con sus perros, cantan o, las menos de las veces, piden dinero o se pelean entre ellos.

Te sientas aquí y [la policía] no te molestas. Aquí uno 'no molesta' y puedes estarte todo el día de puta madre... fresquito. La gente joven es enrollada... si les pides un cigarro, te lo dan (K, 2/10/2017); o:

Hace unos años había un grupito [de sintecho] que estaban todo el día allí [rincón entre Ferlandina y Centre de Documentació] y les dijeron: aquí no podéis estar. Tenían sus colchones, sus bolsas, sus cosas, joder... Más que por el follón que líes no quieren que se te vea mucho. Parece que afeas la plaza (L, 15/09/2017).

Para ellos la plaza era un lugar seguro por la cantidad de gente que hay: "Que te vean, tío, hace que estés más seguro. Con tanta gente nadie te va a joder" (L, 2/10/2017). Su ubicación variaba, aprovechaban los escalones de entrada, algunos rincones menos higiénicos y libres de jóvenes, como entre la entrada del parking y el CEDM; rehuían mezclarse con los grupos de jóvenes y con las familias: "Paso de las 
miraditas raras o de asco" (M, 16/09/2017). Tan habituados al desprecio, ellos mismos buscaban los microlugares de la plaza donde difícilmente podrán mezclarse con el resto.

Cinco: 'Skaters'. Siendo el más llamativo y el que conforma la imagen más duradera de la plaza, el colectivo de los skaters ha conquistado la plaza (Figura 10), ya que su apropiación supedita la apropiación del resto de colectivos: "Siempre está lleno de skaters. Sólo se oyen ruedas" (N, 3/10/2017). La plaza se ha convertido en un lugar de referencia en el mundo del skate:

Que no sean skate parks, las más conocidas son Plaça Universitat y la Barceloneta... pero este es el sitio mejor conectado... Está a cinco minutos de la L1 [línea de metro]... y tiene un montón de cosas [pavimento liso, escaleras, bordillos, etc.] para practicar (0, 3/10/2017).

Los múltiples desniveles de la plaza se prestan a poner a prueba su destreza; en la zona central, los skaters se dedicaban a ensayar flips, hardflips y pop shove its y desviaban los peatones; en las rampas y escaleras, y cuando los guardas de seguridad no los veían, probaban kickturns, ollies, grinds, lidpslide, etc., y en ocasiones se grababan. "La plaza tiene de todo para practicar... para todos los niveles. Te encuentras de todo, cracks y aficionados. Es un sitio cojonudo" (P, 8/09/2017). Durante la etnografía distinguimos ocho grupos recurrentes de skaters, todos de distintas nacionalidad, distinta procedencia dentro de la ciudad y distinto nivel de pericia con el skate. Por las mañanas los grupos de skate se caracterizaban por ser muy jóvenes y principiantes. Por las tardes, los grupos expertos, en los que dominaba una estética pseudoamericana, tomaban paulatinamente la plaza; unos con las escaleras de Montalegre, otros circulan de la entrada del parking hasta el extremo contrario de la plaza; otros, más mayores (entrados en la treintena), se apoyaban contra la fachada del CEDM. Durante las tardes, con una territorialización muy fuerte, no había rincón libre de skaters. Por los saludos y los intercambios lingüísticos en un tosco inglés la plaza parecía el lugar de encuentro de una práctica que les entusiasmaba. Su práctica desviaba por completo los usos programados; de la funcionalidad de acceso al museo y al pasaje entre zonas, se pasaba a un conjunto de elementos en los que se podía disfrutar la ciudad como si de un half pipe se tratara.

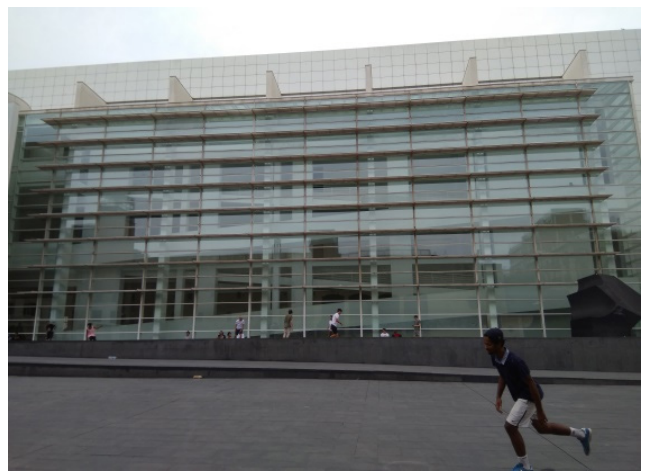

Figura 4 - MACBA. Fuente: Elaboración propia (2017).

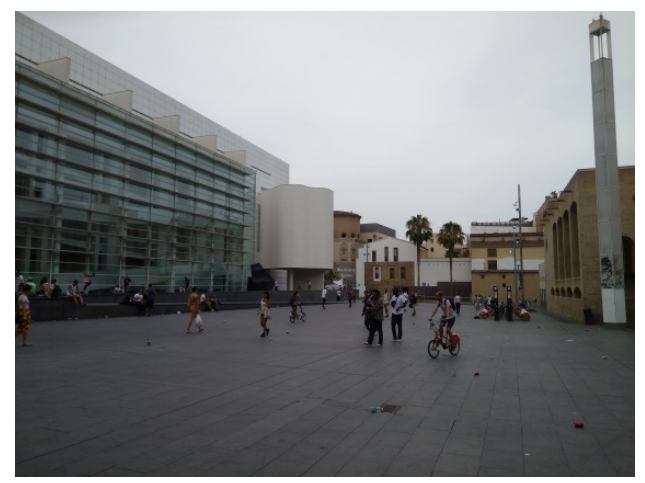

Figura 5 - Flujo constante. Fuente: Elaboración propia (2017). 


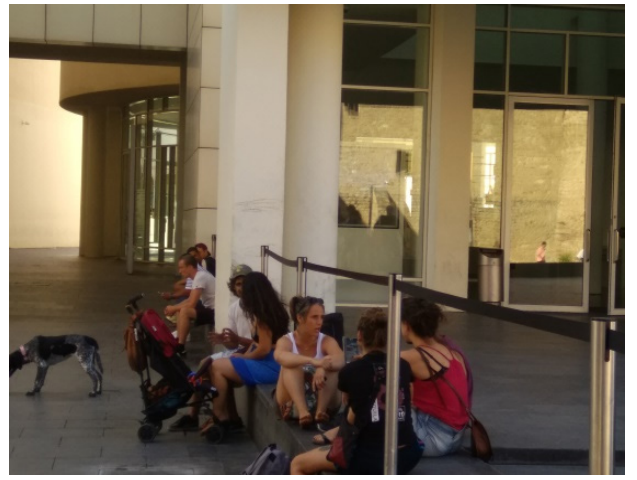

Figura 6 - Familias. Fuente: Elaboración propia (2017).

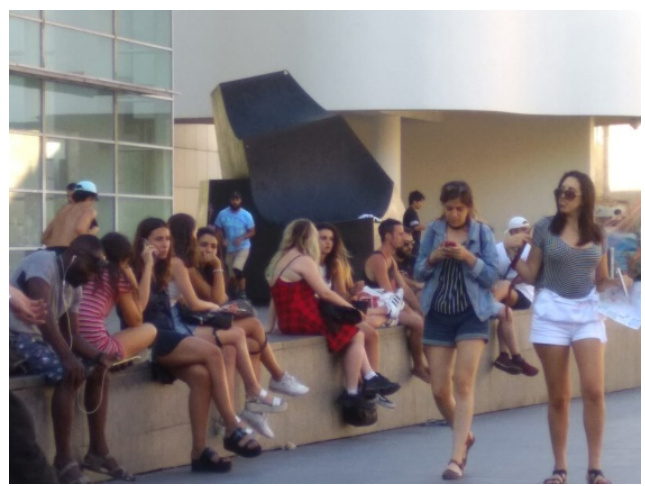

Figura 7 - Jóvenes. Fuente: Elaboración propia (2017).

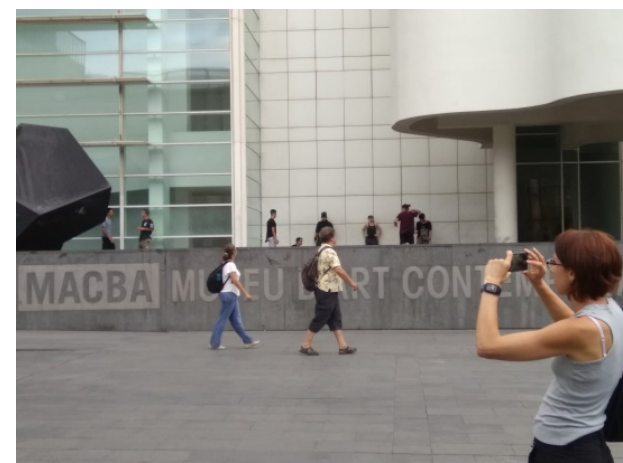

Figura 8 - Turistas. Fuente: Elaboración propia (2017).

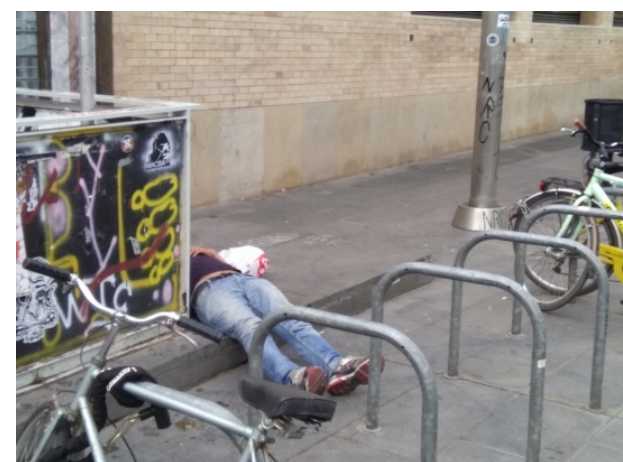

Figura 9 - Sintecho. Fuente: Elaboración propia (2017). 


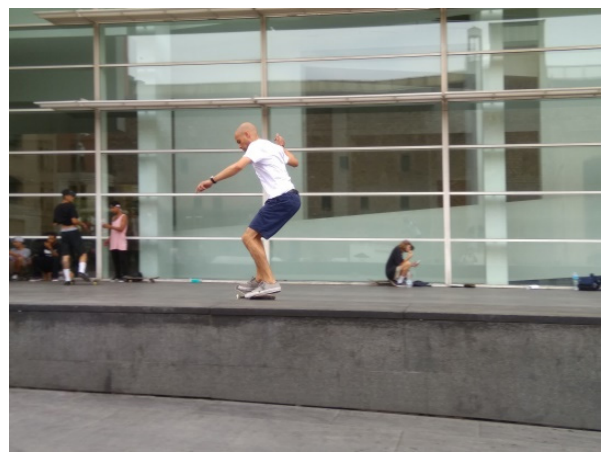

Figura 10 - Skaters. Fuente: Elaboración propia (2017).

Las prácticas cotidianas producen sin capitalizar, semejante al acto de lectura (Certeau, 2000), y en el nivel más superficial del análisis, las apropiaciones que observamos expresaban dos hechos:

1. necesidades de un espacio acorde a sus deseos; $y$

2. resistencias a un cierto discurso de ciudad.

Respecto a (1), a pesar de su dotación insuficiente, la plaza era el único lugar donde los ciudadanos en su heterogeneidad podían dar rienda suelta a necesidades espaciales concretas: las familias de un lugar de juego, los skaters de una superficie y unos desniveles en los que ponerse a prueba, los jóvenes de lugar de reunión amplio y de diversión, los sintecho de protección y tranquilidad, y los turistas de descanso. Los desvíos propuestos por los cinco colectivos demostraban la capacidad adaptativa de los ciudadanos a espacios adversos que, por la propia utilización diaria, se transforman simbólicamente hasta alcanzar un cierto acomode a las necesidades y deseos; hecho que nos demuestra que el espacio es mucho más que su dimensión física y mental: es una cuestión de vida (Soja, 1996).

La plaza es fea, muy fea. No tiene casi de nada. Siempre hay mucha gente. Pero es lo único que tenemos por aquí si queremos una plaza. No está hecha para venir a disfrutarla, pero, digo yo, tendremos que tener una plaza ¿no? (R, 9/09/2017).

En tanto plasmación existencial, la necesidad espacial siempre hallaba, en el caso que nos ocupa, un lugar donde realizarse total o parcialmente; una vida puede inscribirse en el espacio público porque, como Lefebvre (1974) anticipó, lo urbano no consiste en latencias que se disuelven en la irrealización, sino que es puro devenir, una actualización-siempre-en-curso.

Estas necesidades se transformaban en inesperadas apropiaciones de la Plaça dels Àngels que suponían al tiempo (2): una réplica lúdica, desenfada e irreflexiva al 'Modelo Barcelona'.

En Barcelona siempre quieren ir a la última, ser los más modernos. Pero ¿y los que vivimos aquí? Necesito una plaza para llevar a mis hijos. A veces pienso que se olvidan de los que vivimos aquí y hacen la ciudad para los de fuera (S, 9/09/2017).

Esta resistencia es lo que Rose (2002) denomina una 'resistencia involuntaria' [unintentional resistence], elusiva, no adscrita a deseos o intereses conscientes, fruto de un cúmulo contingente y desorganizado de relaciones que multiplican el espacio al reescribir su imaginación geográfica. Así, la vida cotidiana que tenía lugar en la Plaça dels Àngels desdecía el discurso urbano y transgredía los usos proyectados y el determinismo arquitectónico. Día a día registrábamos cómo se ponía en cuestión los funcionamientos preconcebidos y el concepto de 'vida en la ciudad'. En contra de la mercantilización de su valor de uso, la plaza fue reclamada en tanto espacio de deseo y de posibilidad, reflejando las necesidades nada marginales de los ciudadanos que ninguna concepción urbanística, por muy contrapuesta que fuera, podía extinguir. Los ritmos urbanos se inscribían de continuo en la plaza. A través de la informalidad y de la creatividad espacial, los practicantes territorializaban el espacio público en distintos contextos sociales que reinventaban la plaza. 
Las necesidades y las resistencias (Figura 11) estaban allí, delante nuestro; la Plaça dels Àngels hacía las veces de escenario. Podríamos afirmar que el deseo amolda, hasta cierto punto, la ciudad.

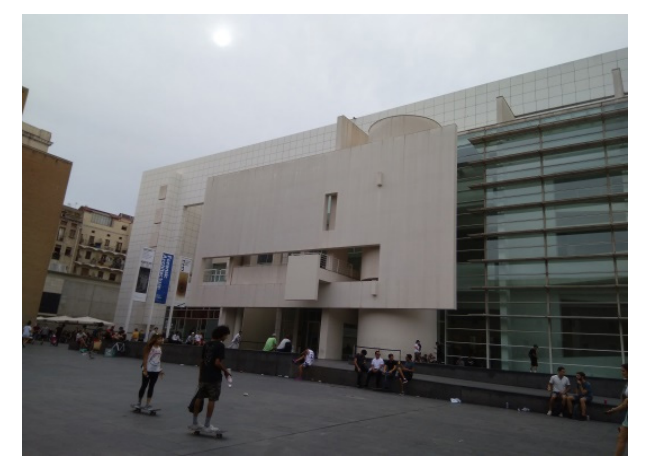

Figura 11 - Apropiaciones. Fuente: Elaboración propia (2017).

Enfatizamos: 'hasta cierto punto', pues es en la propia observación de la apropiación de los colectivos donde encontramos sus principales limitaciones.

La misma territorialidad nos llevó a un nuevo nivel del análisis con nuevas reflexiones menos luminosa. De toda apropiación, asevera Lefebvre (1974), surgen nuevos significados más allá de los originalmente planeados y ésta, en conjunto, suele mostrarse como una yuxtaposición, como un collage. En nuestro caso, esto se traducía a cinco colectivos, cinco formas de entender la plaza, cinco formas de apropiar el espacio delimitándolo en cinco tipos de territorialidad. Para Dematteis (2004), la territorialidad se trata de una mediación simbólica, cognoscitiva y práctica entre la sociedad y la materialidad propia del territorio donde se habita, vive y produce. Y estamos de acuerdo, del mismo modo que la necesidad y la resistencia toman la plaza, la dimensión política y física de la plaza castra la apropiación total y la articulación de una respuesta espacial contundente. Durante la etnografía nos apercibimos que los cinco colectivos se rozaban espacialmente, con fricciones y conflictos muy aislados -"A veces, los skaters molestan mucho. Que se vayan a otro sitio" (T,14/09/2017); "Los vagabundos siempre están pidiendo cigarros" (U, 25/09/2017)-, pero apenas se relacionaban entre ellos. Las apropiaciones espaciales delimitaban territorios de uso casi exclusivo para cada colectivo, donde la co-presencia y la convivencia, por muy efímera que pudiera llegar a ser, se excluía como posibilidad. Puesto que las apropiaciones comportaban exclusiones, algunas necesidades se veían desplazadas a espacios públicos adyacentes, tales como Els Jardins de Torres i Clavé o la Plaça de les Caramelles. Actividades como los juegos de pelota (fútbol y básquet) o que requieren de un espacio público dotado de elementos heterogéneos (escondite, jugar con los columpios, etc.).

Aquí todo el mundo va a lo suyo. Aunque los frikis del skate [los skaters] ocupan un buen trozo de plaza, la gente se pone dónde puede. Unos ahí [las familias], los vagabundos allí... Con los amigos [estudiantes] nos tiramos aquí en el suelo... Es nuestro rincón (V, 9/09/2017).

La plaza estaba férreamente territorializada, cada colectivo estaba cerrado, ensimismado en su propia actividad. Sin puntos de encuentro ni intersecciones, la plaza se presentaba como una sucesión de fragmentos de necesidades y resistencias que no coagulaban en un significado general. Al contrario de lo que sugiere la bibliografía (Amendola, 2000; Gehl, 2011), la respuesta ciudadana a través de la apropiación no se articulaba en una respuesta unitaria a un discurso urbanístico -el 'Modelo Barcelona'que reduce las posibilidades de lo urbano, sino que se contentaba con ser en una sucesión de pequeñas réplicas coyunturalmente coincidentes que evidenciaban los límites de dicho discurso, pero sin capacidad de ofrecer una alternativa. El uso por el que fue planificada y construida la plaza no se respetaba, eso resulta evidente, porque la ciudadanía tenía otras necesidades que ese urbanismo no lograba colmar, pero esa misma manifestación de la insuficiencia no revertía, ni revierte en propuestas rotundas ni en sugerencias que exciten la imaginación geográfica. 
¿Podemos afirmar que las apropiaciones observadas devolvían la virtualidad de lo urbano al espacio público y su carácter revolucionario, se trataba de un espacio liberado? No. Si para Arendt lo público implica la relación entre semejantes, pero también con el Otro, pues lo urbano, superando el terror al desconocimiento recíproco, se despliega en relación con lo extranjero y lo diferente (Amendola, 2000), las apropiaciones en la Plaça dels Àngels fallaban al convocar la totalidad de la comunidad, al construir la construcción de una memoria colectiva. Tal vez, si nos conformásemos con el pensamiento débil [pensiero debole] (Viano, 1985), estas necesidades inscritas contra la dotación espacial y estas réplicas aisladas contra un modelo urbano determinístico nos bastarían. Pero no es el caso. Diríase que se contentan con vivir en los intersticios de un discurso urbanístico que es capaz de apropiarse discursivamente de su apropiación espacial.

De fano, del griego phaínein (alumbrar, mostrar, hacer visible, evidenciar), las cinco tipos de apropiaciones de la Plaça dels Àngels manifiestan la incapacidad ciudadana de articular respuestas efectivas, corrosivas para con los modos y modelos urbanísticos que prescinden de la vida para configurar y ordenar el espacio. Las causas, podemos aventurarnos a conjeturar, pueden ser diversas; clasificaríamos estas posibles causes en:

3. Causa urbanística: la dimensión física de la plaza puede apropiarse a través del uso del espacio pero no modificarse en sus atributos básicos, pues ésta, en lo esencial, sigue permaneciendo igual; este hecho limita el espectro de lo posible a unas apropiaciones superficiales, irrisorias y poco significativas socialmente hablando, que, incluso, podrían llegar a ser anticipadas por los propias urbanistas para otros usos;

4. Causa discursiva: el 'Modelo Barcelona' ha promovido una visión concreta de la ciudad al tiempo que ha sabido apropiarse de actividades y desvíos no originalmente planificados -los skaters, la marginalidad cool y cualquier elemento que, al decir de Sennett (2018), pueda caracterizarse dentro de o caricaturizarse como la dimensión dionisíaca de la ciudad- que enriquecen y refuerzan la modernidad que propugna;

5. Causa ética: el contacto con el otro suscita a partes iguales fascinación y desconfianza (Cixous, 2011), pero sin un interés y una voluntad expresa de vencer las diferencias -el abismo entre los cuerpos y los sentires- y sin encontrar recoprocidades que acaben por establecer lazos de entendimiento, toda aproximación a ese Ajeno y Extraño será un acto frustrado y, por extensión, la creación una comunidad o de un colectivo una posibilidad abolida.

Estas tres causas aquí inferidas pueden ayudar, en investigaciones futuras, a ir más allá de la conceptualización inicial de Lefebvre y profundizar en el límite manifiesto de toda apropiación urbana. Dispersas, sin objetivo, escasamente efectivas, superficiales, y por ende sin consecuencias, las apropiaciones, aunque expresen necesidades y descontentos, diagnostican por así decir los males, mas no infieren la cura. El límite de la apropiación lefebvriana manifiesta la lucha por el derecho a la ciudad, pero resulta a todas luces insuficiente para ganarla.

\section{Conclusiones}

¿Está muerto el espacio público? No de momento.

El espacio público, sostiene Kern (2008), es a un tiempo último reducto no comercializado de la ciudad y la última oportunidad de expresión puramente social. Y nuestra investigación no ha hecho más que confirmarlo. El espacio público sigue reteniendo su potencia original de (1) inscribir necesidades no contempladas inicialmente por el urbanismo -lugar de deseo- y de manifestar respuestas inconscientes y fragmentadas -lienzo de la sociedad-. El urbanismo propagandístico, deliberadamente no participativo y saturado de mistificaciones que enmascaran las posibilidades de la vida en la ciudad (Merrifield, 2002), sigue teniendo en el espacio público esa resistencia irreductible. 
La aparición de lo desviado, una manifestación de lo que Soja (1996) denominaba 'mundos reales e imaginarios' [real-and-imagined worlds], puede contribuir a una evaluación crítica de los discursos y modelos urbanos. Y de hecho, así hemos visto, la Plaça dels Àngels es una manifestación que, cotidianamente y a través de las apropiaciones de los cinco colectivos, recuerda la insuficiencia del 'Modelo Barcelona' para responder las exigencias de lo urbano. Mientras se practique la apropiación, la monofuncionalidad no tendrá lugar en el espacio público.

Sin embargo, si las apropiaciones muestran los límites del urbanismo, la territorialidad autista de los colectivos pone en evidencia los límites de la propia apropiación. Por muy inconsciente, lúdica y desviada que se presente, la réplica carece de fuerza efectiva para propiciar reflexiones a mayor escala. Tres pueden ser las causas que limitan la fuerza renovadora de la apropiación como concepto: urbanísticas, discursivas y éticas.

Sólo si se supera el límite de la apropiación, los imaginarios urbanos tendrán cabida real en la ciudad. Sólo si la respuesta vence la distancia entre las diferencias de los colectivos, podrá elevarse como alternativa. Hagamos nuestras las palabras de Lévinas (1971): el contacto con el otro es la antesala de lo inesperado.

\section{Agradecimientos}

\section{A A. Casellas.}

\section{Referencias}

Abrahao, S. L. (2016). Appropriation and political expression in urban public spaces. Revista Brasileira Estudios Urbanos Regionais, 18(2), 291-303. http://dx.doi.org/10.22296/2317-1529.2016v18n2p291.

Agar, M. (1996). The professional stranger: an informal introduction to ethnography. Nueva York: Academic Press. Amendola, G. (2000). La ciudad postmoderna. Madrid: Celeste Ediciones.

Andrés Sarasa, J. L. (1991). Imágenes del espacio urbano y prácticas comerciales en la ciudad de Murcia. Murcia: Cámara Oficial de Comercio, Industria y Navegación de Murcia.

Ascher, F. (2004). Los nuevos principios del urbanismo: el fin de las ciudades no está a la orden del día. Madrid: Alianza Editorial.

Augé, M. (1993). Los “No lugares", espacios del anonimato: una antropología de la sobremodernidad. Barcelona: Gedisa Editorial.

Barcelona. (1990). Pla Especial d'Ordenació del Conjunt de la Casa de Caritat. Barcelona: Ajuntament de Barcelona.

Barcelona. Foment de Ciutat Vella S.A. - FOCIVESA. (2006). Modificació del PERI del Raval a l'entorn de la Plaça dels Àngels. Barcelona: Ajuntament de Barcelona.

Bassand, M. (1990). Urbanization: appropriations of space and culture. Nueva York: City University of New York.

Benach, N. (1993). Producción de imagen en la Barcelona del 92. Estudios Geográficos, 212, 483-505.

Benach, N. (1997). Ciutat i producció d'imatge: Barcelona 1979-1992 (Tesis doctoral). Universitat de Barcelona, Barcelona.

Benach, N., \& Tello, R. (2004). En los intersticios de la renovación: estrategias de transformación del espacio y flujos de población en Barcelona. Revija za Geografijo, 3, 93-114.

Bohigas, O. (1986). Reconstrucción de Barcelona. Barcelona: Gráfiques Aleu.

Borja, J. (2004). Los derechos en la globalización y el derecho a la ciudad. Madrid: Fundación Alternativas. 
Capel, H. (2005). El modelo Barcelona: Un examen crítico. Barcelona: Ediciones del Serbal.

Carmona, M. (2010). Contemporary public space: critique and classification. Part one: critique. Journal of Urban Design, 15(1), 123-148. http://dx.doi.org/10.1080/13574800903435651.

Casellas, A. (2006). Las limitaciones del 'Modelo Barcelona': una lectura desde urban regime analysis. Documents d'Analisi Geografica, 48, 61-81.

Certeau, M. (2000). La invención de lo cotidiano: artes de hacer. México: Universidad Iberoamericana.

Cixous, H. (2011). Volleys of humanity: essays 1972-2009. Edinburgo: Edinburgh University Press. http://dx.doi.org/10.3366/edinburgh/9780748639038.003.0016.

Chaplin, S., \& Holding, E. (Eds.) (1998). Consuming architecture. Londres: Architectural Design.

Choay, F. (2014). L'urbanisme, utopies et realités. París: Points.

Davis, M. (1990). City of quartz: excavating the future of Los Angeles. New York: Verso.

Dematteis, G. (2004). En la encrucijada de la territorialidad urbana. In Á. Martin Ramos (Ed.), Lo urbano en 20 autores contemporáneos. Barcelona: Edicions UPC.

Duneier, M. (1999). Sidewalk. New York: Farrar, Strauss and Giroux.

Gehl, J. (2011). Life between buildings: using public space. Washington: Island Press.

Glaser, B. G., \& Strauss, A. L. (1967). The discovery of grounded theory: strategies for qualitative research. Chicago: Aldine.

Hammersley, M., \& Atkinson, P. (2007). Ethnography: principles in practice. Londres: Routledge. http://dx.doi.org/10.4324/9780203944769.

Harvey, D. (1973). Social justice and the city. Baltimore: Johns Hopkins University Press.

Harvey, D. (2008). The right to the city. New Left Review, 53, 23-40.

Hiernaux, D., \& Lindón, A. (1996). El concepto de espacio y el análisis regional. Secuencia, 25, 89-109.

Iveson, K. (1998). Putting the public back into public space. Urban Policy and Research, 16(1), 21-23. http://dx.doi.org/10.1080/08111149808727745.

Joseph, I. (1988). El transeunte y el espacio público urbano: ensayo sobre la dispersión del espacio público. Buenos Aires: Gedisa.

Kern, K. (2008). Heterotopia of the theme park street. In M. Dehane, \& L. Cauter (Eds.), Heterotopia and the city: public space in a postcivil society (pp. 105-115). London: Routledge.

Lefebvre, H. (1968). Le droit à la ville. París: Seuil.

Lefebvre, H. (1973). Espace et politique: le droit à la ville II. París: Anthropos.

Lefebvre, H. (1974). La production de l'espace. París: Anthropos.

Lévinas, E. (1971). Totalité et infini: essai sur l'extériorité. París: Livre de Poche.

Lynch, K. (1960). The image of the city. Massachusetts: MIT.

Madanipour, A. (1999). What are the design and development of public spaces significant for cities? Environment \& Planning B, 26(6), 879-891. http://dx.doi.org/10.1068/b260879.

Margier, A. (2017). Cohabiter l'espace public: conflits d'appropriation et rapports de pouvoir à Montréal et Paris. Rennes: PUR.

Massey, D. (2005). For space. Londres: Sage.

Merrifield, A. (2002). Guest editorial: Seattle, Quebec, Genoa: Après le Déluge... Henri Lefebvre. Environment and Planning. D, Society \& Space, 20(2), 127-134. http://dx.doi.org/10.1068/d2002ed. 
Mierzejewska, L. (2011). Appropriation of public urban space as an effect of privatization and globalization. Quaestiones Geographicae, 30(4), 39-46. http://dx.doi.org/10.2478/v10117-011-0036-7.

Montaner, J. M. (2003). Repensar Barcelona. Barcelona: Edicions UPC.

Morente, F. (2018). De la acumulación a la apropiación: una reflexión acerca del espacio público en la ciudad contemporánea. urbe. Revista Brasileira de Gestão Urbana, 10(3), 650-662. http://dx.doi.org/10.1590/21753369.010.003.ao12.

Ostermann, F. O., \& Timpf, S. (2009). Use and appropriation of space in urban public parks. Geographica Helvetica, 64(1), 30-36. http://dx.doi.org/10.5194/gh-64-30-2009.

Paquot, T. (2015). L'espace public. París: La Découverte.

Parkinson, J. R. (2012). Democracy \& public space: the physical sites of democratic performance. Oxford: Oxford University Press. http://dx.doi.org/10.1093/acprof:osobl/9780199214563.001.0001.

Pétonnet, C. (1982). L'observation flotante. l'example d'un cimetière parisien. L'Homme, 22(4), 37-47. http://dx.doi.org/10.3406/hom.1982.368323.

Pujades, J. J. (2002). Barcelona, ciutat oberta: transformacions urbanes, participació ciutadana i cultures de control al barri del Raval. Revista d'Etnologia de Catalunya, 21, 114-131.

Rancière, J. (2015). La partage du sensible: esthètique et politique. París: La Fabrique Éditions.

Reventós Gil, B. (2007). Patrimonios incómodos para la imagen que Barcelona ofrece al mundo. Pasos: Revista de Turismo y Patrimonio Cultural, 5(3), 287-305. http://dx.doi.org/10.25145/j.pasos.2007.05.021.

Rius, J., \& Sánchez-Befando, M. V. (2015). Modelo Barcelona y política cultural: usos y abusos de la cultura por parte de un modelo emprendedor de desarrollo local. EURE. Revista Latinoamericana de Estudios Urbano Regionales, 41(122), 103-123. http://dx.doi.org/10.4067/S0250-71612015000100005.

Rose, M. (2002). The seduction of resistance: power, politics, and a performative style of systems. Environment and Planning. D, Society \& Space, 20(4), 384-400. http://dx.doi.org/10.1068/d262t.

Rouet, G. (2014). Mobilisations citoyennes dans l'espace public. París: L'Harmattan.

Saint-Blancat, C., \& Cancellieri, A. (2014). From invisibility to visibility? The appropriation of public space through a religious ritual: the Filipino procession of santacruzan in Padua, Italy. Social \& Cultural Geography, 15(6), 645663. http://dx.doi.org/10.1080/14649365.2013.879494.

Schneider, J. (2013). Measuring transportation at a human scale: an intercept survey approach to capture pedestrian activity. Journal of Transport and Land Use, 6(3), 43-59. http://dx.doi.org/10.5198/jtlu.v6i3.311.

Seidman, I. (2006). Interviewing as qualitative research: a guide for reseachers in education and the social sciences. Nueva York: Teachers College Press.

Sennett, R. (2018). Building and dwelling: ethics for the city. New York: Farrar Strauss \& Giroux.

Soja, E. W. (1996). Thirdspace: journeys to Los Angeles and other real-and-imagined places. Nueva York: WileyBlackwell.

Sorkin, M. (Ed.). (1992). Variations on a theme park: the new American city and the end of public space. Nueva York: Hill \& Wang.

Staeheli, L. A., \& Mitchell, D. (2007). Locating the public in research and practice. Progress in Human Geography, 31(6), 792-811. http://dx.doi.org/10.1177/0309132507083509.

Subirats, J., \& Rius, J. (1998). Del Xino al Raval: cultura i transformació social a la Barcelona Central. Barcelona: Centre de Cultura Contemporànea de Barcelona, Urbanitats.

Subirós, J. (1999). Estrategias culturales y renovación urbana. Barcelona: Aula Barcelona.

Tapia, M. (2007). Superposición de realidades: colectivos minoritarios en el espacio público de Ciutat Vella, Barcelona (Tesis doctoral). Universitat Politècnica de Catalunya, Escola Tècnica Superior d'Arquitectura, Barcelona. 
Trier, J. (Eds.) (2014). Detournement as pedagogical praxis. Rotterdam: Sense Publishers. http://dx.doi.org/10.1007/978-94-6209-800-8.

Viano, C. A. (1985). Va'pensiero: il carattere della filosofía italiana contemporánea. Torino: Einaudi.

Vidal, T., \& Pol, E. (2005). La apropiación del espacio: una propuesta teórica para comprender la vinculación entre las personas y los lugares. Anuario de Psicología, 36, 281-297.

Wittmer, E. (2017). Aménager les espace publics: mettre en place le processus. París: Territorial Éditions.

Yin, R. K. (2011). Qualitative research from start to finish. New York: Guilford Press.

Zukin, S. (1995). The cultures of cities. Oxford: Blackwell.

Editor: Rodrigo Firmino

Recibido: Jul. 31, 2018

Aprobado: Nov. 01, 2018 